\title{
INTERPOLATION OF SIGNALS BY GENERALIZED PIECEWISE-LINEAR MULTIPLE GENERATORS
}

\author{
Koichi ICHIGE ${ }^{\dagger}$, Thierry BLU ${ }^{\dagger \dagger}$ and Michael UNSER ${ }^{\dagger \dagger}$ \\ $\dagger$ Department of Electrical and Computer Engineering, Yokohama National University \\ Yokohama 240-8501, Japan. E-mail: koichi@ynu.ac.jp \\ ${ }^{\dagger \dagger}$ Biomedical Imaging Group, FSTI/IOA, Swiss Federal Institute of Technology Lausanne \\ CH-1015 Lausanne, Switzerland. E-mail: \{thierry.blu, michael.unser\}@epfl.ch
}

\begin{abstract}
This paper presents an interpolation method based on shifted versions of two piecewise linear generators, which provides approximation order 2 like usual piecewise-linear interpolation; i.e., this method is able to represent the constant and the ramp exactly.

Our interpolation is characterized by two real parameters: $\tau$, the location of the generators, and $\alpha$, related to their dissymmetry. By varying these parameters, we show that it is possible to optimize the quality of the approximation, independently of the function to interpolate. We recover the optimal value of shifted-linear interpolation $(\tau=0.21$ and $\alpha=1$ ) which requires IIR prefiltering, but we also find a new configuration $(\tau=0.21$ and $\alpha=0.58$ ) which reaches almost the same quality, while requiring FIR filtering only. This new solution is able to greatly reduce the amount of Gibbs oscillations generated in the shifted-linear interpolation scheme.

We validate our finding by computing the PSNR of the difference between multi-rotated images and their original version.
\end{abstract}

\section{INTRODUCTION}

Interpolation is one of the standard operations in digital signal/image processing $[1,2]$. Among the multitude of algorithms that have been proposed, piecewise-linear interpolation is still the method of choice for many applications due to its low computational cost. The down-side of the standard algorithm is the poor quality of the results; piecewise-linear interpolation typically introduces blurring and streaking artifacts that can be disturbing visually, especially in image processing applications.

Recently, two of the present authors have proposed shiftedlinear interpolation [3] which greatly improves the quality of reconstructed signals/images while preserving the low computational complexity of piecewise-linear interpolation. While it clearly outperforms the classical algorithm in terms of quality, it has one negative side effect; namely, the introduction of a Gibbs-like phenomenon (oscillations) when interpolating step-like functions, as mentioned in [3]. The presence of these oscillations tends to increase the dynamic range of the output image; this effect is especially noticeable in successive interpolation experiments such as multiple image rotations.

Both standard and shifted linear interpolation methods employ wavelet-like basis functions that are shifted replicates of a single generator: the "tent" function, which has a central axis of symmetry. In principle, this type of representation can be generalized by introducing multiple generating functions which are not necessarily symmetric anymore. However, to be acceptable from an approximation theoretic point of view, the representation should preserve the "partition of unity" property; more generally, it should allow for the perfect reconstruction of linear polynomials [4]. The question that we address in this paper is whether or not there are advantages to switching to representations with multiple generators for the task of signal/image interpolation.

In this paper, we will concentrate on a piecewise-linear model that uses two generators instead of one: this is still reasonably simple, but already constitutes a radical departure from the traditional approach. We will evaluate the approach with respect to the specific task of signal/image interpolation. First, we will solve the corresponding interpolation problem and propose a filterbank algorithm for the efficient determination of the expansion coefficients of the model. Second, we will apply the method to image interpolation and compare its various versions with the traditional approach. This will allow us to determine the optimal parameters values for best performance according to two criteria: (1) maximum PSNR (Peak Signal-to-Noise Ratio), and (2) minimization of the Gibbs-like oscillations. With respect to this last item, we found that the prefiltering (decomposition) scheme could be implemented by FIR filters for a whole range of parameter values, which essentially gets rid of this problem. 


\section{PRELIMINARIES}

The standard and shifted linear interpolations are summarized in this section.

Consider a sequence of samples $f[n]=f(n T)$ sampled from a function $f(x)$ with the sampling interval $T$. The standard linear interpolation gives an interpolated function $f_{T}(x)$ as

$$
f_{T}(x)=\sum_{n \in \boldsymbol{Z}} f[n] \varphi\left(\frac{x}{T}-n\right),
$$

which simply corresponds to connecting two neighboring samples with a straight line. The basis function $\varphi$ is given as: $\varphi(x)=1-|x|$ for $|x| \leq 1$ and $\varphi(x)=0$ for $|x|>1$.

The shifted linear interpolation [3] gives the interpolated function as

$$
f_{T}(x)=\sum_{n \in \boldsymbol{Z}} c[n] \varphi\left(\frac{x}{T}-n-\tau\right)
$$

where $\tau$ denotes the shift parameter; the coefficients $\{c[n]\}_{n \in Z}$ are derived recursively as

$$
c[n]=-\frac{\tau}{1-\tau} c[n-1]+\frac{1}{1-\tau} f[n],
$$

which leads to an IIR filter implementation. The optimum value of $\tau$ is approximately 0.21 [3]. These basis functions are depicted in Fig.1 (a) and (b).

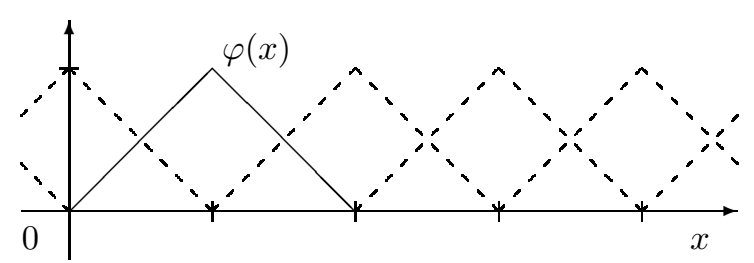

(a) standard linear interpolation

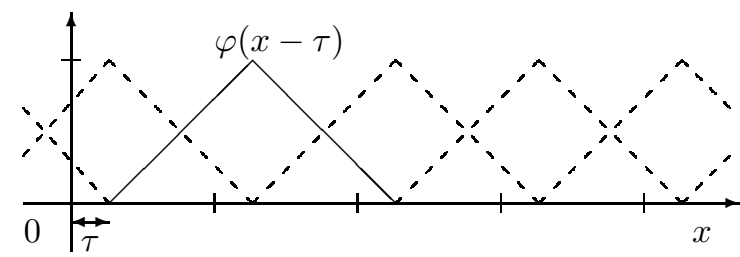

(b) shifted-linear interpolation

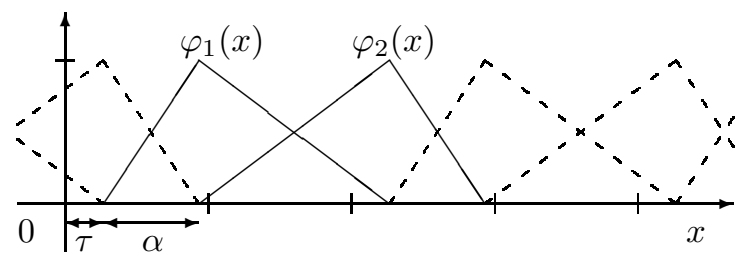

(c) proposed method

Fig. 1. Basis functions.

\section{GENERALIZED PIECEWISE-LINEAR MULTIPLE GENERATORS}

The concept of the shifted-linear interpolation can be further generalized by introducing dissymmetric basis functions. Here we employ two dissymmetric generating functions $\varphi_{1}$ and $\varphi_{2}$, mirror to each other as in Fig.1(c), i.e.

$$
\begin{aligned}
& \varphi_{1}(x)= \begin{cases}\frac{x-\tau}{\alpha}, & \tau \leq x<\tau+\alpha \\
\frac{2+\tau-x}{2-\alpha}, & \tau+\alpha \leq x<\tau+2 \\
0, & \text { otherwise }\end{cases} \\
& \varphi_{2}(x)= \begin{cases}\frac{x-\tau-\alpha}{2-\alpha}, & \tau+\alpha \leq x<\tau+2 \\
\frac{\tau+\alpha+2-x}{\alpha}, & \tau+2 \leq x<\tau+\alpha+2 \\
0, & \text { otherwise }\end{cases}
\end{aligned}
$$

The signal is reconstructed by

$$
f[n]=\sum_{k \in \boldsymbol{Z}} c_{1}[k] \varphi_{1}[n-2 k]+\sum_{k \in \boldsymbol{Z}} c_{2}[k] \varphi_{2}[n-2 k] .
$$

The parameters $\tau$ and $\alpha$ correspond to the shift and the dissymmetry, respectively. For any $x \in \boldsymbol{R}$, the basis functions satisfy the partition of unity:

$$
\sum_{n \in \boldsymbol{Z}}\left\{\varphi_{1}(x-2 n)+\varphi_{2}(x-2 n)\right\}=1 .
$$

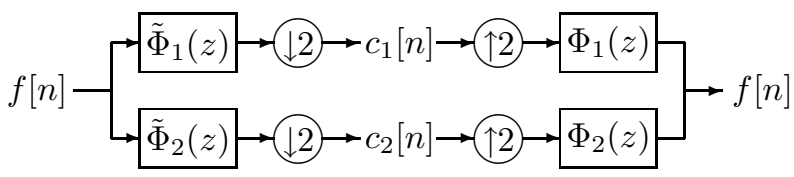

Fig. 2. Filterbank construction of the proposed scheme.

If $\tau+\alpha<1$, the coefficients $c_{1}$ and $c_{2}$ can be derived simply by

$$
\begin{aligned}
& c_{1}[n]=(2-\tau-\alpha) f[2 n+1]-(1-\tau-\alpha) f[2 n+2], \\
& c_{2}[n]=(1+\alpha) f[2 n+2]-\alpha f[2 n+1],
\end{aligned}
$$

which enables the FIR filter implementation of the decomposition process. The whole process can be drawn as Fig.2 in a filterbank form; the transfer functions of the prefilters $\tilde{\Phi}_{1}$ and $\tilde{\Phi}_{2}$ for the decomposition are

$$
\begin{aligned}
& \tilde{\Phi}_{1}(z)=(2-\tau-\alpha) z-(1-\tau-\alpha) z^{2}, \\
& \tilde{\Phi}_{2}(z)=(1+\alpha) z^{2}-\alpha z
\end{aligned}
$$

If $\tau+\alpha \geq 1$, the prefilters $\tilde{\Phi}_{1}$ and $\tilde{\Phi}_{2}$ become

$\tilde{\Phi}_{1}(z)=\frac{\tau(2-\tau-\alpha) z+(2-\tau)(1-\tau-\alpha)}{(1-\alpha)(2-\tau-\alpha)+\alpha(1-\tau-\alpha) z^{-2}}$,

$\tilde{\Phi}_{2}(z)=\frac{(2-\tau)(1-\alpha) z^{2}-\tau \alpha z}{(1-\alpha)(2-\tau-\alpha)+\alpha(1-\tau-\alpha) z^{-2}} ;$ 
they are implemented by Forward/Backward IIR filtering.

\section{SIMULATION}

We now evaluate the proposed scheme of piecewise-linear multiple generators through some simulations.

Similarly to [3], we first evaluate the functions through 15 successive image rotations by $24^{\circ}$. The target images are as shown in Figs.4-6(a) which have different characteristics: "Lena" mainly has low frequency components, "Baboon" contains every frequency component and "Particles" is a binary image to be regarded as an extreme example.

We tested various combinations of values of the parameters $\tau$ and $\alpha$ for those three images to see which combination is the most suitable. Fig.7 depicts the behavior of PSNRs for various values of the parameters $\tau$ and $\alpha$. From Fig.7, we can see that the case of the shifted-linear interpolation ( $\tau=0.21, \alpha=0$ ) was the best for all of three images with respect to PSNR. Indeed the reconstructed images are sharp as shown in Figs.4-6(c), much more precise than in the case of standard linear interpolation in Figs.4-6(b).

In Fig.7, we also find that there is another local optimum PSNR around $\tau=0.21$ and $\alpha=0.58$. The PSNR here is somewhat lower than that in the shifted-linear case, but the reconstructed images have almost the same quality as shown in Figs.4-6(c),(d). The maximum and minimum values of the reconstructed images covers a wider range as in the shifted-linear case, while they are closer to the original in the local optimum case as listed in Table 1. As we see in Fig.5(c), there are some white and black pixels like impulse noise. The values of these pixels are very far from the original range, as suggested from the data in Table 1.

Table 1. Maximum and minimum values of the original and rotated images

\begin{tabular}{|c|c|c|c|}
\hline & Lena & Baboon & Particles \\
\cline { 2 - 4 } & Min/Max & Min/Max & Min/Max \\
\hline Original & $0 / 255$ & $6 / 227$ & $0 / 255$ \\
\hline Linear & $0 / 255$ & $6 / 227$ & $0 / 255$ \\
\hline Shifted-Linear & $-105 / 369$ & $-31 / 384$ & $-420 / 1015$ \\
\hline $\begin{array}{c}\text { Proposed } \\
(\tau=0.21, \alpha=0.58)\end{array}$ & $-50 / 343$ & $-5 / 245$ & $-200 / 410$ \\
\hline
\end{tabular}

The wide range in the shifted-linear interpolation is a consequence of the Gibbs-like oscillations when interpolating step-like functions. Figure 3 shows the result of interpolating a unit step function. Shifted-linear interpolation causes oscillation for several steps. In the local optimum case ( $\tau=0.21$ and $\alpha=0.58$ ), there are two possible behaviors (a) and (b) due to two different basis functions. The oscillation in (a) is smaller than that in Shifted-linear, and the case (b) does not make any oscillation. They lead to the smaller range in Table 1.

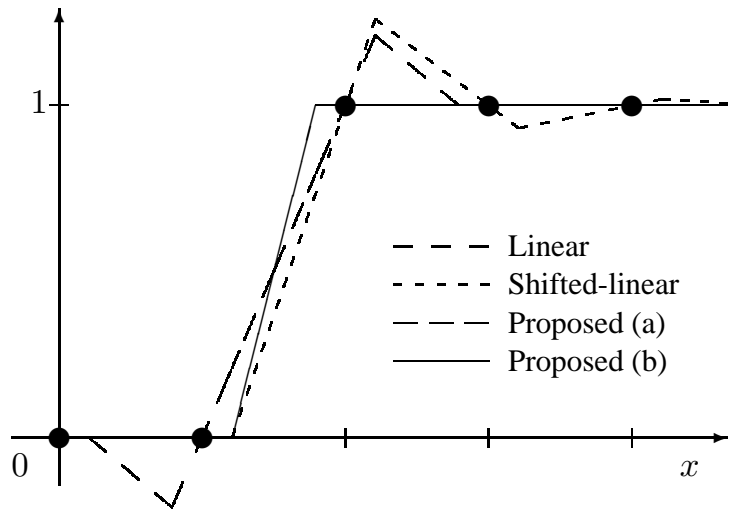

Fig. 3. Interpolation of a unit step function.

The computation time for the proposed scheme is almost the same as that for the shifted-linear interpolation, provided the processes for $c_{1}$ and $c_{2}$ in Fig.2 are executed in parallel.

\section{CONCLUDING REMARKS}

In this paper, a concept of generalized piecewise-linear multiple generators and its applications have been presented. The present dissymmetric basis functions can be regarded as generalized piecewise linear interpolators. We developed a scheme of decomposition and reconstruction of signals/images by those functions in a form of a filterbank. Then the scheme was evaluated through computer simulation of digital image rotation.

The error in interpolation and the optimum values of the parameters must be further studied mathematically. Moreover, the case of three (or more) functions should be investigated in the future.

\section{REFERENCES}

[1] P. Thévenaz, T. Blu and M. Unser, "Image Interpolation and Resampling," in Handbook of Medical Imaging, Processing and Analysis, I.N. Bankman Ed., pp. 393-420. Academic Press, San Diego, CA, U.S.A, 2000.

[2] P. Thévenaz, T. Blu and M. Unser, "Interpolation Revisited," IEEE Trans. Med. Imag., vol. 19, no. 7, pp. 739-758, July 2000.

[3] T. Blu, P. Thévenaz and M. Unser, "How A Simple Shift Can Significantly Improve The Performance of Linear Interpolation," Proc. IEEE Int'l Conf. on Image Proc., pp. III.377-III.380, Rochester, NY, U.S.A, Sep. 2002.

[4] G. Strang, T. Nguyen, Wavelet and Filter Banks, Wellesley Cambridge Pr., MA, U.S.A., 1996. 


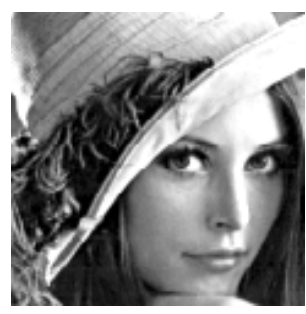

(a) Original

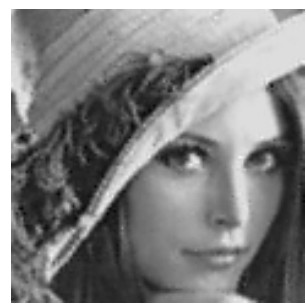

(c) Shifted-Linear

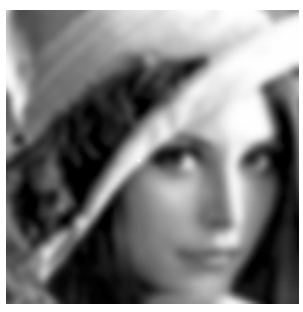

(b) Linear

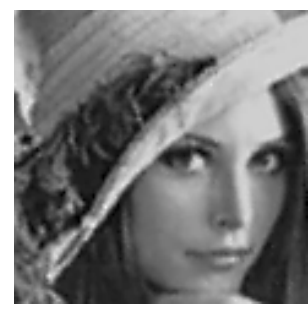

(d) $\tau=0.21, \alpha=0.58$
Fig. 4. Rotated Images of "Lena"

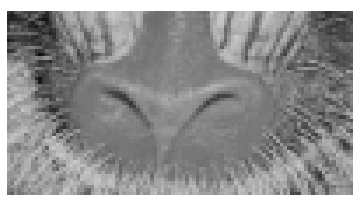

(a) Original

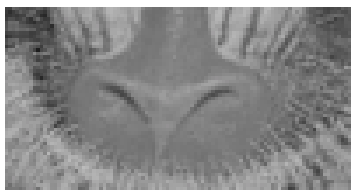

(c) Shifted-Linear

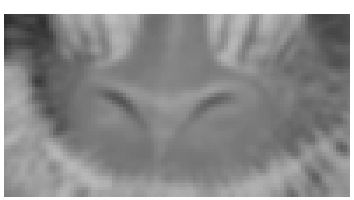

(b) Linear

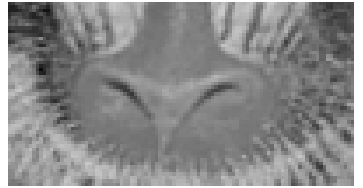

(d) $\tau=0.21, \alpha=0.58$
Fig. 5. Rotated Images of "Baboon"

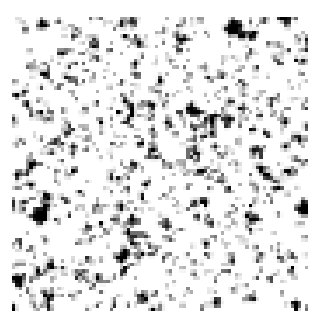

(a) Original

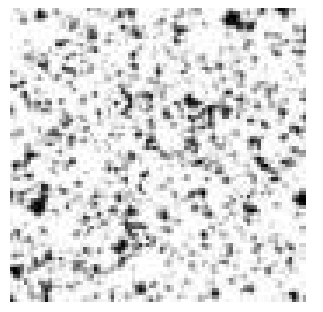

(c) Shifted-Linear

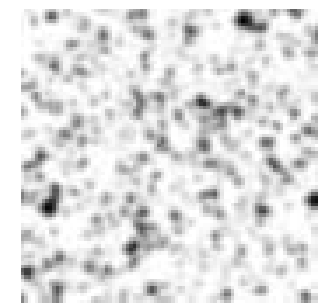

(b) Linear

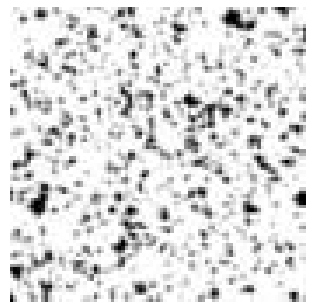

(d) $\tau=0.21, \alpha=0.58$
Fig. 6. Rotated Images of "Particles"

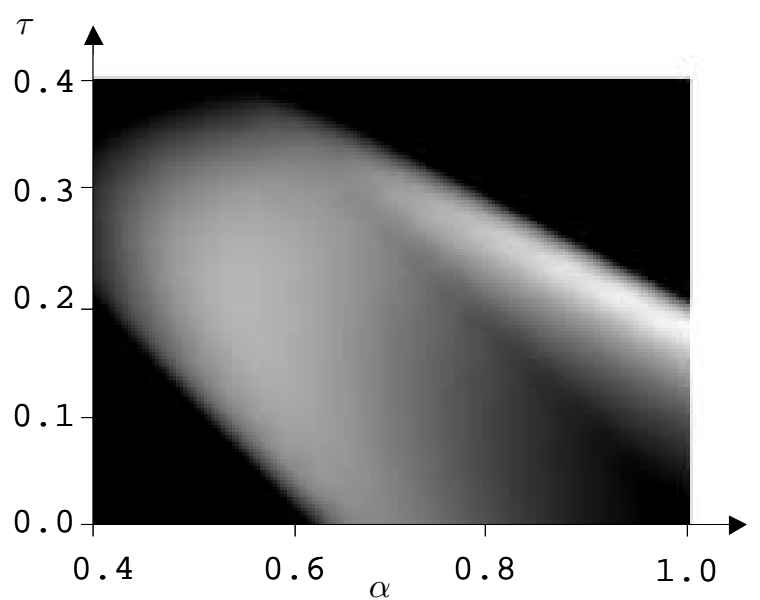

(a) Lena; white $=28 \mathrm{~dB}$, black $=22 \mathrm{~dB}$ or less

Linear: 22.0dB, Shifted-Linear: $28.1 \mathrm{~dB}$, Proposed: $27.6 \mathrm{~dB}$

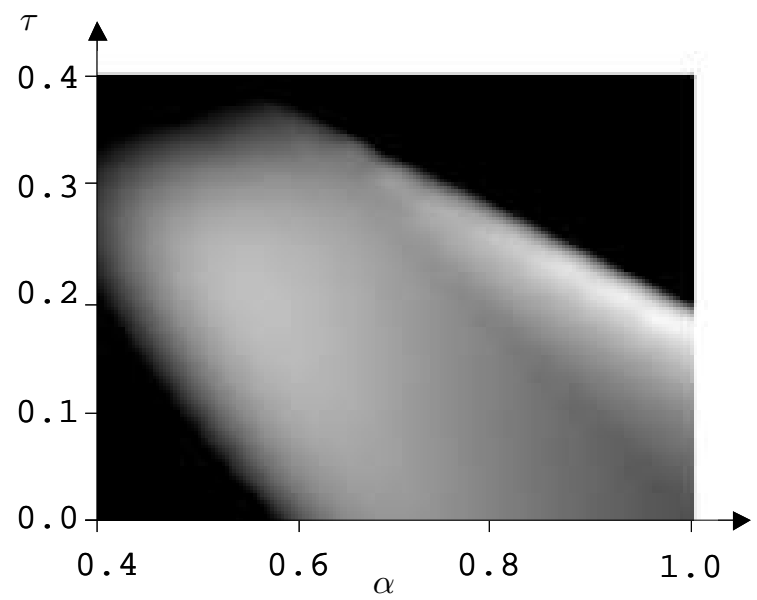

(b) Baboon; white $=18.5 \mathrm{~dB}$, black $=15 \mathrm{~dB}$ or less Linear: 16.2dB, Shifted-Linear: 18.6dB, Proposed: 18.0dB

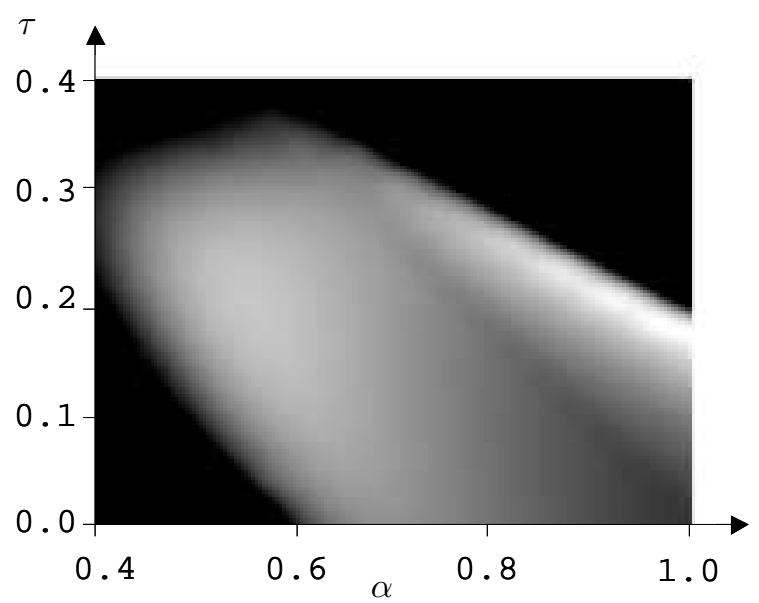

(c) Particles; white $=13.5 \mathrm{~dB}$, black $=10 \mathrm{~dB}$ or less Linear: 10.6dB, Shifted-Linear: 13.3dB, Proposed: $12.8 \mathrm{~dB}$

Fig. 7. PSNRs of the rotated images for various combinations of parameters $\alpha$ and $\tau$. Note that the optimum solution is obtained in white regions. 